\title{
Long-term treatment effects in chronic myeloid leukemia
}

\author{
Apollos Besse $^{1,2}$, Thomas Lepoutre ${ }^{2,1}$, and Samuel Bernard ${ }^{1,2}$ \\ 1 Univ Lyon, Université Claude Bernard Lyon 1, CNRS UMR 5208, Institut Camille \\ Jordan, 43 blvd. du 11 novembre 1918, F-69622 Villeurbanne cedex, France \\ apollos.besse@inria.fr, \\ 2 INRIA
}

\begin{abstract}
We propose and analyze a simplified version of a partial differential equation (PDE) model for chronic myeloid leukemia (CML) derived from an agent-based model proposed by Roeder et al. This model describes the proliferation and differentiation of leukemic stem cells in the bone marrow and the effect of the drug Imatinib on these cells. We first simplify the PDE model by noting that most of the dynamics occurs in a subspace of the original $2 \mathrm{D}$ state space. Then we determine the dominant eigenvalue of the corresponding linearized system that controls the long-term behavior of solutions. We mathematically show a non-monotonous dependence of the dominant eigenvalue with respect to treatment dose, with the existence of a unique minimal negative eigenvalue. In terms of CML treatment, this shows that there is a unique dose that maximizes the decay rate of the CML tumor load over long time scales. Moreover this unique dose is lower than the dose that maximizes the initial tumor load decay. Numerical simulations of the full model confirm that this phenomenon is not an artifact of the simplification. Therefore, while optimal asymptotic dosage might not be the best one at short time scales, our results raise interesting perspectives in terms of strategies for achieving and improving long-term deep response.
\end{abstract}

Keywords: Chronic Myeloid Leukemia, Renewal model, Dominant eigenvalue, Structured population dynamic

\section{Introduction}

Chronic Myeloid Leukemia is a blood and bone marrow cancer that results in a white blood cells overproduction. This disease is associated with the presence of the Philadelphia chromosome, which is produced by a characteristic chromosomal translocation. This mutation leads to a new gene $B C R-A B L$ instead of the original $A B L$.

The chimeric BCR-ABL gene codes for a tyrosine kinase, a protein that stimulates abnormal white blood cell production, through ill-defined mechanisms (Deininger et al. 2008). Unlike most cancers, CML is not detected by the presence of a solid tumor, but by a blood test in which cancer progress can be estimated 
by measuring the ratio of $\mathrm{BCR}-\mathrm{ABL}$ gene expression compared to $\mathrm{ABL}$ gene expression.

Leukemic cells proliferation process begins in the bone marrow by the activation of quiescent cancer stem cells. Once activated, these non-differentiated cells divide, and then differentiate into blood cells. The small number and the low basal activity of stem cells make any direct characterization of these cells difficult. Once committed for differentiation, leukemic cells will divide over twenty times before being released in the blood. They are then generically called mature cells (actually, in certain phases, immature cells can be released in the blood). Circulating leukemic cells are not dividing anymore, and are cleared from the blood within a few days, so the BCR-ABL/ABL rate represents evolution of the disease in bone marrow, with a delay of few weeks.

The Tyrosine kinase inhibitor (TKI) Imatinib is the first treatment to have specifically targeted the BCR-ABL gene in leukemic cells. It limits the leukemic cells proliferation and promotes apoptosis (cell death) of leukemic cells. The effect of TKI on healthy cells is negligible in case of treatment with standard doses (Graham et al. 2002). TKI treatments have therefore excellent short-term and long-term results (O'Brien et al. 2003). However the disease seems to persist. After stopping treatment, most patients relapse. Yet recent clinical trials have shown that a long TKI treatment duration could cause definitive remission of patient after treatment withdrawal, even with residual leukemic stem cells (Rousselot et al. 2007, Mahon et al. 2010, Preudhomme et al. 2010). In a recent clinical trial, Hehlmann et al. (2014) compared different doses of Imatinib. They have shown that a high-dose treatment leads to a deep response faster than a standard dose. However, some patients do not tolerate Imatinib toxicity. Breccia et al. (2010) have shown that low-dose Imatinib appears effective in patients with intolerance to the standard dose.

Several mathematical models for the dynamics of CML co-exist in the literature. Michor et al. (2005) proposed a model that explains the failure to completely eradicate the disease under TKI treatment by the development of a resistance to these inhibitors in leukemia cells. This model may not allow recovery of the experimental results in case of combined treatments (Foo et al. 2009). Komarova \& Wodarz (2007) proposed a model that mixes the stem cells ability to become quiescent and the development of resistance to treatment. The effect of immune response was studied by Kim et al. (2008b) by adding an immune compartment to the model of Michor et al.. Recently, Clapp et al. (2015) proposed a model that describes interactions between leukemic cells and immune response. They explain clinically obtained oscillations by the competition between immune response and immunosuppression .

Roeder et al. (2006) proposed an agent-based model (ABM) in which the fate of each cell is stochastically determined according to its characteristics and its environment. The model describes the competition of leukemic stem cells versus healthy stem cells in the bone marrow, and the effect of TKI on this competition. Leukemic and healthy stem cells are separated into two states, proliferative and quiescent, structured by their progress in the cell cycle and 
by their ability to differentiate. In addition, it is assumed that TKI treatment does not affect healthy cells. Glauche et al. (2012) used this model to study combined treatment effect, and found that the combination of a continuous dose of TKI coupled with an intermittent dose of Interferon (a drug used to treat CML before the development of TKIs) shows the best efficacy while limiting side effects. Kim et al. (2008c) proposed to replace the ABM model by a system of deterministic difference equations in order to reduce complexity. A PDE version of the original ABM model was also proposed independently by the same authors (Kim et al. 2008a) and by Roeder et al. (2009). Stability analysis of the resulting model, under further simplifications, has been proposed by Doumic-Jauffret et al. (2010).

In this paper, we propose a simplification of the PDE model presented by Kim et al. (2008a). It is close to the model studied by Doumic-Jauffret et al. (2010). We study asymptotic behavior and convergence speed to eradication of the disease. We show that long-term TKI efficacy, as measured by the asymptotic tumor growth rate, is non-monotonous with respect to dose.

The paper is organized as follows. In section 2, we present the ABM model of Roeder et al. (2006), its PDE version given by Kim et al. (2008a) and the simplification we choose to make. In section 3 , we investigate existence and uniqueness of a disease-free steady state. Then, we analyze the asymptotic behavior of solutions close to this steady state, by searching the linearized system dominant eigenvalue. In section 4 , we study the treatment dose effect on the dominant eigenvalue that controls the asymptotic decay speed of solutions near the disease-free steady state.

\section{PDE reduction of the agent-based model}

In this section, we outline the partial differential equations (PDE) version of the ABM model, proposed by Kim et al. (2008a) and summarized by DoumicJauffret et al. (2010).

The reduction of the agent-based model to partial differential equations brings three significant changes: numerical simulations are faster and can process arbitrarily large population densities, solutions are more regular since the PDE model ignores stochastic effects, and mathematical analysis becomes feasible.

\subsection{Description of the PDE model}

The original ABM model describes the dynamics of leukemia in the bone marrow. It is useful to study the competition between leukemic and healthy stem cells population, before differentiation. Leukemic and healthy differentiated and mature cells populations are the model output, and evolve afterwards.

A stem cell is assumed to be able to self-renew when the body needs it, and to differentiate prior to exiting the bone marrow. We distinguish two states in the life of a leukemic or healthy stem cell before differentiation;

- proliferation, 
- quiescence.

The proliferative state of the cell cycle is traditionally divided into four phases: first gap (G1), synthesis (S), second gap (G2) and mitosis (M), at the end of which the cell divides. It is assumed here that the dividing cell gives rise to two identical daughter cells. We separate proliferative cells into two stages: those in the G1 phase (stage 1) and those in S, G2 and M phases (stage 2). Duration of these phases are assumed to be fixed: $c_{1}$ for G1, and $c_{2}$ for S-G2-M. The entire cell cycle duration is then $C=c_{1}+c_{2}$.

Stages 1 and 2 are structured by two variables: the cell cycle progress $c$, and the affinity $x$, a variable that represents the tendency for cells to differentiate.

The cell cycle progress $c$ is assumed to increase linearly as a function of time $(d c / d t=1)$, from 0 to $c_{1}$ in stage 1 , and from 0 to $c_{2}$ in stage 2 . A cell in stage 1 with $c=c_{1}$ directly enters stage 2 with $c=0$. A cell in stage 2 with $c=c_{2}$ divides and creates two cells in stage 1 , with $c=0$.

In the proliferative state, affinity $x$ is assumed to increase linearly with time from 0 to $1\left(d x / d t=\rho_{P}>0\right)$. When a cell reaches $x=1$, it leaves the proliferative state and goes to differentiation.

The quiescent state is assumed to be structured only by affinity $x$, which decreases linearly with time from 1 to $0\left(\frac{d x}{d t}=-\rho_{Q}\right.$ if $\left.0<x \leqslant 1\right)$ and stays at 0 when $x=0(d x / d t=0$ if $x=0)$.

Quiescent cells can be activated and become proliferative with a transition rate $\beta$. They enter the proliferative stage 2 with the same affinity $x$ and are assigned a cell-cycle progress $c=0$. Only proliferative cells in stage 1 can deactivate to quiescence, with a transition rate $\alpha$. They enter the quiescent state with the same affinity $x$.

We will denote $Q=Q(t, x), x \in(0,1]$ the quiescent cell density of affinity $x$ at time $t ; Q^{*}(t)$ the number of quiescent cells of affinity $x=0$ at time $t ; P_{i}(t, x, c)$ the proliferating cell density in stage $i \in\{1,2\}$ that transited from a quiescent state with $x>0$; and $P_{i}^{*}(t, x)$ the cell density in stage $i$ that transited from the quiescent state with $x=0$. The reason to distinguish cells that went through $x=0$ is that they accumulate and induce a singular mass (a Dirac mass) that cannot be modeled as a flux balance.

The transition rates $\alpha$ and $\beta$ depend on the affinity $x$ and on the total amount of quiescent and proliferative cells (leukemic and healthy) in the system, $Q_{t o t}$ and $P_{t o t}$. Transition rates have the functional form:

$$
\alpha\left(x, Q_{t o t}\right)=a^{x} f\left(Q_{t o t} ; \nu\right) \quad \text { and } \quad \beta\left(x, P_{t o t}\right)=a^{1-x} f\left(P_{t o t} ; \nu\right)
$$

where $a$ is a small positive constant, and $f$ is a sigmoidal function whose coefficient $\nu$ depends on the type (healthy or leukemic), the state (quiescent or proliferative) and the treatment used (none or TKI). The function $f$ is positive, decreasing and bounded by 1 (Figure 1):

$$
f(u)=\frac{1}{\nu_{1}+\nu_{2} \exp \left(\nu_{3} \frac{u}{N}\right)}+\nu_{4}
$$

Parameter values are summarized in Table 1. 


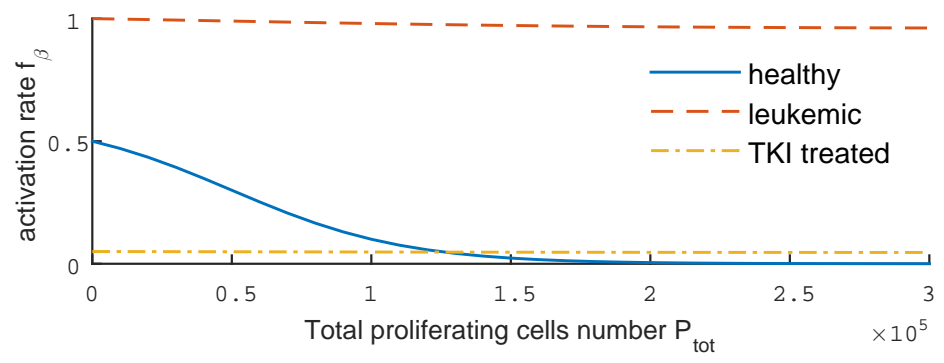

Fig. 1. Examples of transition functions $f_{\beta}$ for three different individuals. The activation rate $\beta$ is given by the formula $\beta\left(x, P_{t o t}\right)=a^{1-x} f_{\beta}\left(P_{t o t}\right)$.

Table 1. Parameter values and descriptions.

\begin{tabular}{|c|c|c|c|c|}
\hline \multicolumn{4}{|c|}{ Param. Healthy CML / CML+TKI Unit } & Description \\
\hline$\xi$ & & $0 / 1$ & & dose effect $(\xi \in[0,+\infty))$ \\
\hline C & 48 & 48 & $\mathrm{~h}$ & cycle duration \\
\hline$c_{1}$ & 16 & 16 & $\mathrm{~h}$ & cycle first phase duration \\
\hline$c_{2}$ & 32 & 32 & $\mathrm{~h}$ & cycle second phase duration \\
\hline$n_{D}$ & 20 & 20 & & differentiated cell division number \\
\hline$t_{D}$ & 20 & 20 & $\mathrm{~d}$ & time to release in blood \\
\hline$t_{M}$ & 8 & 8 & $\mathrm{~d}$ & lifetime of mature cells \\
\hline$a$ & 0.002 & 0.002 & & constant linked to transfer rates \\
\hline$\rho_{P}$ & 0.0078 & 0.0078 & $\mathrm{~h}^{-1}$ & affinity speed for proliferative cells \\
\hline$\rho_{Q}$ & 0.0156 & 0.0156 & $\mathrm{~h}^{-1}$ & affinity speed for quiescent cells \\
\hline$m$ & 0 & $0 / 0.032$ & $\mathrm{~h}^{-1}$ & proliferative cell death rate \\
\hline$P_{\text {tot }}^{e q}$ & 19,689 & 0 & cell & Model IIc disease-free equilibrium \\
\hline$Q_{\text {tot }}^{e q}$ & 94,605 & 0 & cell & Model IIc disease-free equilibrium \\
\hline$N$ & $10^{5}$ & $10^{5}$ & cell & population scaling factor \\
\hline$\nu_{1}^{(\alpha)}$ & 1.9972 & 0.9992 & $\mathrm{~h}$ & transition function (1) \\
\hline$\nu_{2}^{(\alpha)}$ & 0.0028 & 0.0008 & $\mathrm{~h}$ & transition function (1) \\
\hline$\nu_{3}^{(\alpha)}$ & 8.7641 & 9.9558 & & transition function (1) \\
\hline$\nu_{4}^{(\alpha)}$ & 0 & 0 & $\mathrm{~h}^{-1}$ & transition function (1) \\
\hline$\nu_{1}^{(\beta)}$ & 1.6667 & $16.667 / 166.67$ & $\mathrm{~h}$ & transition function (1) \\
\hline$\nu_{2}^{(\beta)}$ & 0.3333 & $8.3333 / 83.333$ & $\mathrm{~h}$ & transition function (1) \\
\hline$\nu_{3}^{(\beta)}$ & 3.2189 & 1.3863 & & transition function (1) \\
\hline$\nu_{4}^{(\beta)}$ & 0 & $0.9600 / 0.9992$ & $\mathrm{~h}^{-1}$ & transition function (1) \\
\hline
\end{tabular}




\subsection{The equations}

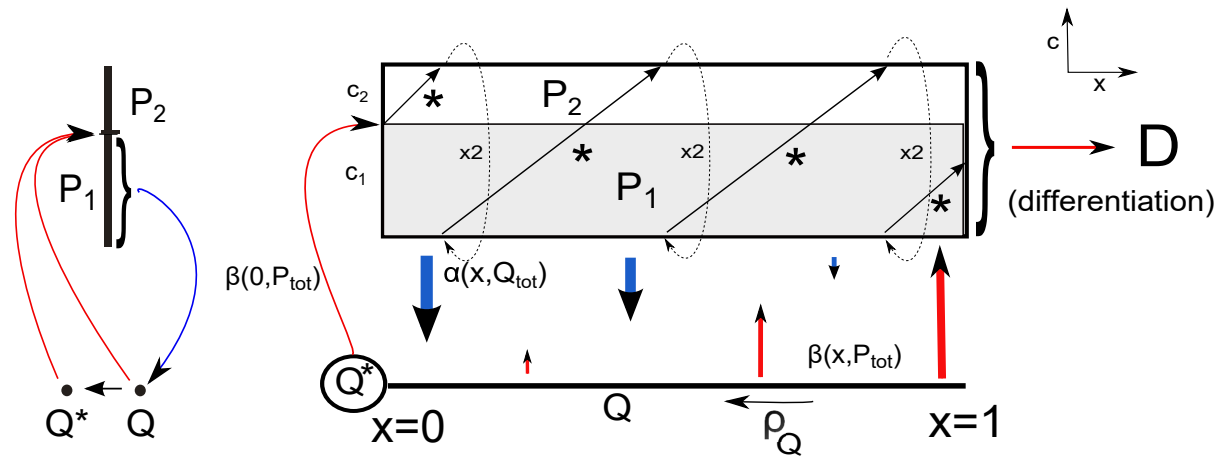

Fig. 2. Scheme of the PDE model. Proliferative and quiescent cell populations are represented on the $2 \mathrm{D}$ space $(x, c)$. On the left is a cut in the direction corresponding to the cycle variable $c$. On the right, diagonal lines represent the place where $*$-cells live. Blue and red arrows represent transition rates between proliferative and quiescent populations.

The densities $P_{1}^{*}(t, x)$ and $P_{2}^{*}(t, x)$ and $\int_{0}^{c_{1}} P_{1}(t, x, c) d c$ represent the same type of quantities, cell densities of affinity $x$. Unlike in $P_{1}$ and $P_{2}$, in $P_{1}^{*}$ and $P_{2}^{*}$ the cell cycle variable is directly determined by the equation $c^{*}=c_{1}+\frac{x^{*}}{\rho_{P}}$ $\bmod C$.

This means that the density $P_{2}^{*}(x, t)$ is only defined on the union of intervals on $x$ :

$$
I_{2}=\left[0, \rho_{P} c_{2}\right) \cup\left[\rho_{P} C, \rho_{P}\left(C+c_{2}\right)\right) \cup\left[2 \rho_{P} C, \rho_{P}\left(2 C+c_{2}\right)\right),
$$

and the density $P_{1}^{*}(t, x)$ is only defined on the union of intervals:

$$
I_{1}=\left[\rho_{P} c_{2}, \rho_{P} C\right) \cup\left[\rho_{P}\left(C+c_{2}\right), 2 \rho_{P} C\right) \cup\left[\rho_{P}\left(2 C+c_{2}\right), 1\right] .
$$

Proliferative and quiescent cells are available in two versions, one for healthy cells and one for leukemia cells. They evolve simultaneously in the bone marrow, and interact only through the dependence of transfer rates $\alpha$ and $\beta$ on $P_{t o t}$ and $Q_{t o t}$. When needed, variables and parameters for leukemic or healthy cells will be indexed by $l$ or $h$ to avoid confusion. When not indexed, variables and parameters are supposed to address both cases. 
Model I (full PDE):

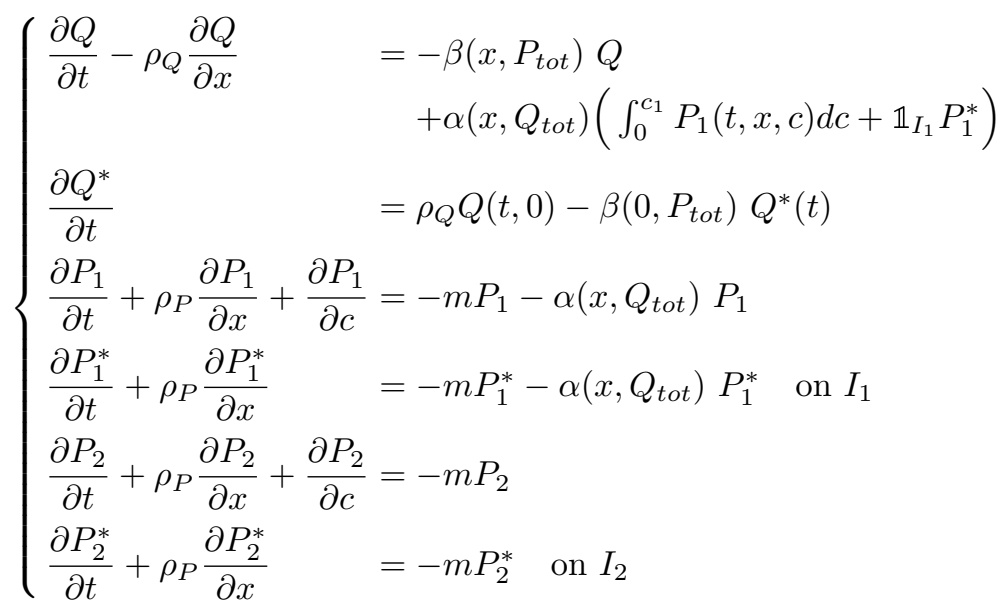

The boundary conditions:

$$
\left\{\begin{array}{l}
Q(t, 1)=0 \\
\rho_{P} P_{2}(t, x, 0)=\rho_{P} P_{1}\left(t, x, c_{1}\right)+\rho_{Q} \beta\left(x, P_{t o t}\right) Q(t, x) \\
\rho_{P} P_{2}^{*}(t, 0)=\beta\left(0, P_{t o t}\right) Q^{*}(t) \\
P_{2}^{*}\left(t, \rho_{P} k C\right)=P_{1}^{*}\left(t, \rho_{P} k C\right), \quad k=1,2, \\
P_{1}(t, x, 0)=2 P_{2}\left(t, x, c_{2}\right) \\
P_{1}^{*}\left(t, \rho_{P}\left(c_{2}+k C\right)\right)=2 P_{2}^{*}\left(t, \rho_{P}\left(c_{2}+k C\right)\right), \quad k=0,1,2 .
\end{array}\right.
$$

where:

$$
\begin{aligned}
Q_{t o t}(t)= & \int_{0}^{1} Q_{l}(t, x) d x+Q_{l}^{*}(t)+\int_{0}^{1} Q_{h}(t, x) d x+Q_{h}^{*}(t), \\
P_{t o t}(t)= & \int_{0}^{1} \int_{0}^{c_{2}} P_{2, l}(t, x, c) d c d x+\int_{0}^{1} \int_{0}^{c_{1}} P_{1, l}(t, x, c) d c d x \\
& +\int_{0}^{1} P_{2, l}^{*}(t, x) d x+\int_{0}^{1} P_{1, l}^{*}(t, x) d x \\
& +\int_{0}^{1} \int_{0}^{c_{2}} P_{2, h}(t, x, c) d c d x+\int_{0}^{1} \int_{0}^{c_{1}} P_{1, h}(t, x, c) d c d x \\
& +\int_{0}^{1} P_{2, h}^{*}(t, x) d x+\int_{0}^{1} P_{1, h}^{*}(t, x) d x .
\end{aligned}
$$

The term $\rho_{Q} Q(t, 0)$ in the second equation of Model $\mathbf{I}$ corresponds to the outgoing flux of quiescent cells reaching affinity $x=0$. Numerical solutions of this model are represented in figure 3 . 

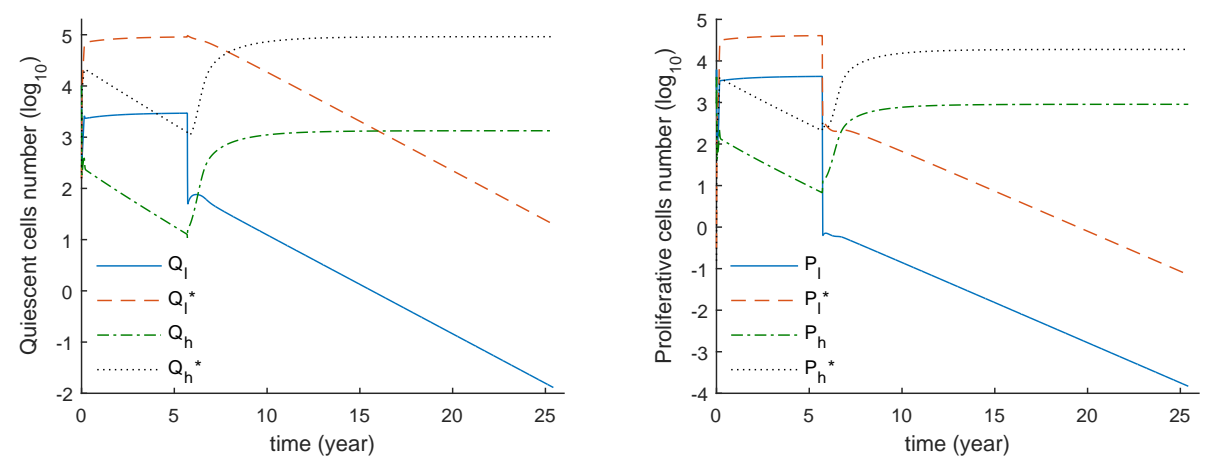

Fig. 3. Quiescent and proliferating cells progress over time in $\log _{10}$ scale, in the case of a TKI treatment (introduced when BCR-ABL/ABL level reaches 99\%), according to Model I. Proliferative populations are the sum of corresponding population in phase 1 and $2\left(P=\bar{P}_{1}+\bar{P}_{2}\right)$. Parameters used are those from Table 1 , and initial conditions are chosen to represent the genesis of leukemia (few leukemic cells and a lot of healthy cells), but are hidden by the high speed of the transient behavior.

Differentiated cells are counted in the flux $D$ defined by:

$$
D(t)=\rho_{P}\left(\int_{0}^{c_{2}} P_{2}(t, 1, c) d c+\int_{0}^{c_{1}} P_{1}(t, 1, c) d c+P_{1}^{*}(t, 1)\right)
$$

Once differentiated, cells divide a fixed number of times $n_{D}$ in $t_{D}$ hours before maturing and being detectable in the blood. The life duration of mature cells in the blood is $t_{M}$ (see supplementary table 1 in the article by Roeder et al. (2006)). The amounts of healthy and leukemic mature blood cells are thus given by the formula, where time unit is in hours:

$$
M(t)=2^{n_{D}} \int_{t-t_{D}-t_{M}}^{t-t_{D}} D(u) d u
$$

Finally, BCR-ABL/ABL ratio representing the disease progress in the body, is given by:

$$
\tau(t)=\frac{M_{l}(t)}{M_{l}(t)+2 M_{h}(t)} .
$$

This formula takes into account the fact that there are two alleles of the ABL gene in healthy cells, and only one allele of the BCR-ABL gene in leukemia cells (Roeder et al. 2006, Glauche et al. 2012).

Coefficients $\rho_{P}$ and $\rho_{Q}$, estimated by Roeder et al. (2006), verify the relationship $\rho_{Q}=1.95 \rho_{P}$. To simplify the numerical scheme, it is assumed that $\rho_{Q}=2 \rho_{P}$. This assumption greatly simplifies the numerical scheme and speed up computer simulations. This change in parameter values affects the asymptotic speed for the simplified model by less than $0.1 \%$ (see Section 3 ). 


\subsection{Simplified model}

Numerically, *-cells represent more than $90 \%$ of the cells in the proliferative state (Figure 3). That phenomenon can be explained by noting that majority of exchanges between quiescent and proliferating cells takes place for small values of affinity. In fact, quiescent cells that activate before entering in $Q^{*}$, are likely to differentiate after cycling (i.e do not return in quiescence), so they are negligible in the long-term dynamic. This observation suggests that the effect of transition rate $\beta(x>0)$ could be neglected. This means that quiescent cells necessarily go through $Q^{*}$ before they activate, and that populations $P_{1}$ and $P_{2}$ vanish. In practice, this simplification leads to a decrease in the number of differentiated and mature cells (because $*$-cells differentiate less frequently than non $*$-cells), but does not change the qualitative behavior of the BCR-ABL/ABL ratio.

Doumic-Jauffret et al. (2010) made the same simplification but added others hypothesis. Their aim was to study the stability of the PDE model and its dependence on parameters. Our aim is to study the dependence of the asymptotic behavior on the treatment parameters, and we show that this analytic study is still feasible with our weaker simplification.

Setting $\beta(x>0)=0$ and $P_{1}(t, x, c)=P_{2}(t, x, c)=0$ leads to the simplified model, denoted Model IIa

Model IIa:

$$
\begin{cases}\frac{\partial Q}{\partial t}-\rho_{Q} \frac{\partial Q}{\partial x} & =\tilde{\alpha}\left(x, Q_{t o t}\right) P^{*} \\ \frac{\partial Q^{*}}{\partial t} & =\rho_{Q} Q(t, 0)-\beta\left(0, P_{t o t}\right) Q^{*}(t) \\ \frac{\partial P_{1}^{*}}{\partial t}+\rho_{P} \frac{\partial P_{1}^{*}}{\partial x} & =-m P_{1}^{*}-\alpha\left(x, Q_{t o t}\right) P_{1}^{*} \\ \frac{\partial P_{2}^{*}}{\partial t}+\rho_{P} \frac{\partial P_{2}^{*}}{\partial x} & =-m P_{2}^{*}\end{cases}
$$

The boundary conditions:

$$
\left\{\begin{array}{l}
Q(t, 1)=0 \\
\rho_{P} P_{2}^{*}(t, 0)=\beta\left(0, P_{t o t}\right) Q^{*}(t) \\
P_{2}^{*}\left(t, \rho_{P} k C\right)=P_{1}^{*}\left(t, \rho_{P} k C\right), \quad k=1,2, \\
P_{1}^{*}\left(t, \rho_{P}\left(c_{2}+k C\right)\right)=2 P_{2}^{*}\left(t, \rho_{P}\left(c_{2}+k C\right)\right), \quad k=0,1,2 .
\end{array}\right.
$$

Numerical solutions of this model are represented in Figure 4.

The simplification lead to a much simpler system (7) because of the exact knowledge of initial affinity $x=0$.

In order to reduce the number of populations and equations in this model, we merge populations $P_{1}^{*}$ and $P_{2}^{*}$ into a single population $P^{*}$, defined for all $x \in[0,1]$ by $P^{*}(t, x)=\mathbb{1}_{I_{1}}(x) P_{1}^{*}(t, x)+\mathbb{1}_{I_{2}}(c) P_{2}^{*}(t, x)$. To take into account this modification in the model, we introduce the deactivation rate $\tilde{\alpha}$ defined as a 

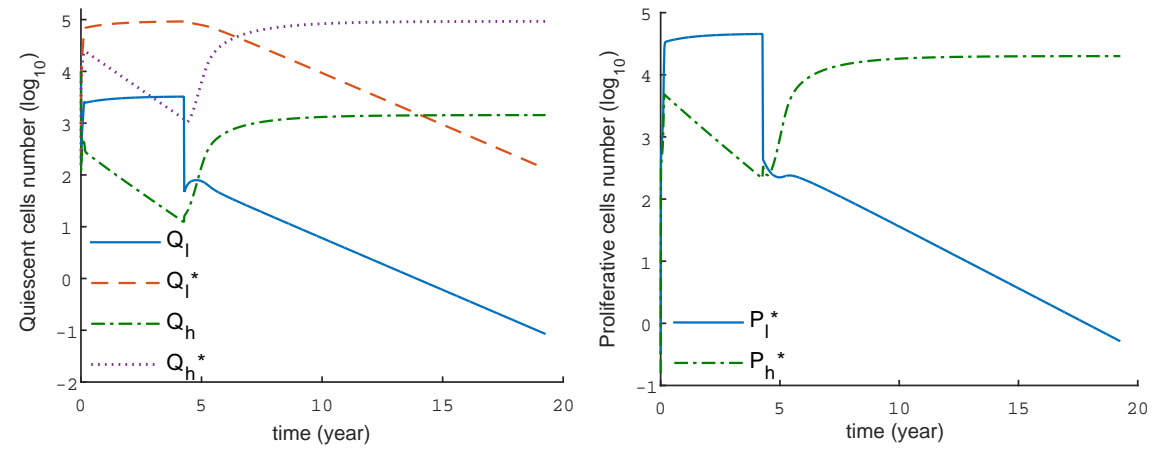

Fig. 4. Quiescent and proliferating cells evolution over time in $\log _{10}$ scale, in the case of a TKI treatment (introduced when BCR-ABL/ABL level reaches 99\%), according to Model IIa. Proliferative populations are the sum of corresponding population in phase 1 and $2\left(P=\bar{P}_{1}+\bar{P}_{2}\right)$. Parameters used are those from Table 1 , and initial conditions are chosen to represent the genesis of leukemia (few leukemic cells and a lot of healthy cells), but are hidden by the high speed of the transient behavior.

piece-wise function of affinity on $[0,1]: \tilde{\alpha}\left(x, Q_{t o t}\right)=\mathbb{1}_{I_{1}}(x) \alpha\left(x, Q_{t o t}\right)$. The model then becomes:

Model IIb:

$$
\begin{cases}\frac{\partial Q}{\partial t}-\rho_{Q} \frac{\partial Q}{\partial x} & =-\beta\left(x, P_{t o t}\right) Q+\tilde{\alpha}\left(x, Q_{t o t}\right) P^{*} \\ \frac{\partial Q^{*}}{\partial t} & =\rho_{Q} Q(t, 0)-\beta\left(0, P_{t o t}\right) Q^{*}(t) \\ \frac{\partial P^{*}}{\partial t}+\rho_{P} \frac{\partial P^{*}}{\partial x} & =-m P^{*}-\tilde{\alpha}\left(x, Q_{t o t}\right) P^{*}\end{cases}
$$

The boundary conditions:

$$
\left\{\begin{array}{l}
Q(t, 1)=0 \\
\rho_{P} P^{*}(t, 0)=\beta\left(0, P_{t o t}\right) Q^{*}(t) \\
P^{*}\left(t, \rho_{P}\left(c_{2}+k C\right)\right)=2 P^{*}\left(t, \rho_{P}\left(c_{2}+k C\right)\right), \quad k=0,1,2 .
\end{array}\right.
$$

The boundary condition at $x=\rho_{P} k C$ is not really needed. For cells in $P^{*}$ the (hidden) cell cycle variable could be directly linked to $x$ by equation $x=\rho_{P}\left(c-c_{1}+k C\right)$, where $k$ is the number of cycles the cell has performed since its entrance in the proliferative compartment. This observation allows us to express the simplified model (7) in an equivalent, but more straightforward manner.

The variable $k$ contains all the information on divisions, which are causing discontinuities of $P^{*}$ at points $x=\rho_{P}\left(c_{2}+k C\right)$. Let us define the quantity 
$\tilde{P}(t, x)$ as

$$
\tilde{P}(t, x)=2^{-k} P^{*}(t, x) \text { if } x+\rho_{P} c_{1} \in\left[\rho_{P} k C, \rho_{P}(k+1) C\right)
$$

The quiescent stem cells density $Q$ and the activation rate $\beta$ stay unchanged, but deactivation rate $\tilde{\alpha}$ depends now on the number of cycles the cell has performed, that is to say the quantity $k=\left\lfloor\frac{x+\rho_{P} c_{1}}{\rho_{P} C}\right\rfloor$.

- We consider the continuous density $\tilde{P}$.

- We denote:

$$
\begin{aligned}
& \chi_{1}=2 \mathbb{1}_{\left[\rho_{P} c_{2}, \rho_{P} C\right]}+4 \mathbb{1}_{\left[\rho_{P}\left(C+c_{2}\right), 2 \rho_{P} C\right]}+8 \mathbb{1}_{\left[\rho_{P}\left(2 C+c_{2}\right), 1\right]}, \\
& \chi_{2}=\mathbb{1}_{\left[0, \rho_{P} c_{2}\right]}+2 \mathbb{1}_{\left[\rho_{P} C, \rho_{P}\left(C+c_{2}\right)\right]}+4 \mathbb{1}_{\left[2 \rho_{P} C, \rho_{P}\left(2 C+c_{2}\right)\right]},
\end{aligned}
$$

- Total amount of proliferating cells are now:

$$
\bar{P}(t)=\int_{0}^{1}\left(\chi_{1}+\chi_{2}\right)(x) \tilde{P}(t, x) d x .
$$

To alleviate the notations, we choose, from now on, to remove the tilde off the density $P$, since there is no possible confusion. This leads to what we will denote Model IIc. Although it is formulated differently, it is technically equivalent to Models IIa and IIb.

\section{Model IIc:}

$$
\left\{\begin{aligned}
\frac{\partial Q}{\partial t}-\rho_{Q} \frac{\partial Q}{\partial x} & =\chi_{1}(x) \tilde{\alpha}\left(x, Q_{t o t}\right) P \\
\frac{\partial Q^{*}}{\partial t} & =\rho_{Q} Q(t, 0)-\beta\left(0, P_{t o t}\right) Q^{*}(t) \\
\frac{\partial P}{\partial t}+\rho_{P} \frac{\partial P}{\partial x} & =-m P-\tilde{\alpha}\left(x, Q_{t o t}\right) P
\end{aligned}\right.
$$

with boundary conditions:

$$
\left\{\begin{array}{l}
Q(t, 1)=0 \\
\rho_{P} P(t, 0)=\beta\left(0, P_{t o t}\right) Q^{*}(t)
\end{array}\right.
$$

The term $\chi_{1}(x) \tilde{\alpha}\left(x, Q_{t o t}\right) P(x)$ in the first equation comes from the fact that divisions are counted in transition rate $\tilde{\alpha}$ through the function $\chi_{1}$, instead of being directly counted in density $P$.

\section{Linear stability analysis of Model IIc}

In this section we analyze the asymptotic behavior of the Model IIc. For sake of simplicity, population $P^{*}$ will be noted $P$, in this section, since there is no ambiguity. In case of an effective treatment, we expect leukemic populations to vanish and healthy to stabilize, as in Figure 4. So, we look for a disease-free steady state $\left(Q_{h}, Q_{h}^{*}, P_{h}, 0,0,0\right)^{T}$. Then, we will seek to obtain information on the behavior of leukemic populations $\left(Q_{l}, Q_{l}^{*}, P_{l}\right)^{T}$ around zero. The system that governs these populations depends on quantities $Q_{t o t}$ and $P_{t o t}$, so it is necessary to study first the nonzero steady state of healthy populations. 


\subsection{Positive steady states of the healthy populations in the absence of leukemic cells}

Here, it is assumed that all leukemic populations vanish. Healthy cells are assumed to behave autonomously. So, we will look for any nonzero stationary solutions for healthy populations. We consider the case where $\alpha_{h}$ and $\beta_{h}$ respectively depend on $\bar{Q}_{h}$ and $\bar{P}_{h}$, and we are looking for time-independent positive solutions. In order to alleviate the notation, we simply note $\beta_{h}=\beta_{h}\left(0, \bar{P}_{h}\right)$, and $\tilde{\alpha}_{h}(x)=\tilde{\alpha}_{h}\left(x, \bar{Q}_{h}\right)$.

The system of equations at equilibrium is reduced to an ordinary differential equation (ODE) system in affinity $x$ :

$$
\left\{\begin{array}{l}
\rho_{Q} \frac{\partial Q}{\partial x}=-\chi_{1}(x) \tilde{\alpha}_{h}(x) P, \\
\rho_{P} \frac{\partial P}{\partial x}=-\tilde{\alpha}_{h}(x) P
\end{array}\right.
$$

with boundary conditions:

$$
\left\{\begin{array}{l}
\beta_{h} Q^{*}=\rho_{Q} Q(0) \\
Q(1)=0 \\
\rho_{P} P(0)=\beta_{h} Q^{*}
\end{array}\right.
$$

The ODE system is solved directly taking into account boundary conditions to yield the solution:

$$
\left\{\begin{array}{l}
P_{h}(x)=\frac{\beta_{h}}{\rho_{P}} Q_{h}^{*} \exp \left(-\int_{0}^{x} \frac{\tilde{\alpha}_{h}(s)}{\rho_{P}} d s\right) \\
Q_{h}(x)=\frac{\beta_{h}}{\rho_{Q}} Q_{h}^{*}\left[1-\int_{0}^{x} \chi_{1}(y) \frac{\tilde{\alpha}_{h}(y)}{\rho_{P}} \exp \left(-\int_{0}^{y} \frac{\tilde{\alpha}_{h}(s)}{\rho_{P}} d s\right) d y\right]
\end{array}\right.
$$

The boundary condition $Q_{h}(x=1)=0$ provides a condition that must be satisfied by the function $x \mapsto \tilde{\alpha}_{h}\left(x, Q_{t o t}\right)$ at equilibrium:

$$
\int_{0}^{1}\left[\chi_{1}(y) \frac{\tilde{\alpha}_{h}\left(y, Q_{t o t}\right)}{\rho_{P}} \exp \left(-\int_{0}^{y} \frac{\tilde{\alpha}_{h}\left(s, Q_{t o t}\right)}{\rho_{P}} d s\right)\right] d y=1
$$

The conditions to obtain a unique positive steady state are summarized in the following proposition.

Proposition 1 We set

$$
\begin{gathered}
J_{1}=\int_{\rho_{P} c_{2}}^{\rho_{P} C} \frac{\tilde{\alpha}_{h}\left(s, Q_{t o t}\right)}{\rho_{P}} d s, \\
J_{2}=\int_{\rho_{P}\left(C+c_{2}\right)}^{2 \rho_{P} C} \frac{\tilde{\alpha}_{h}\left(s, Q_{t o t}\right)}{\rho_{P}} d s,
\end{gathered}
$$




$$
J_{3}=\int_{\rho_{P}\left(2 C+c_{2}\right)}^{1} \frac{\tilde{\alpha}_{h}\left(s, Q_{t o t}\right)}{\rho_{P}} d s
$$

and

$$
\varphi: Q_{t o t} \mapsto e^{J_{1}+J_{2}+J_{3}}+2 e^{J_{2}+J_{3}}+4 e^{J_{3}}-8 .
$$

If $f_{\alpha_{h}}(\infty)=0$ and $\varphi(0)>0$, equation (8) has a unique strictly positive solution.

Proof. Equation 8 can be expressed as

$$
\begin{aligned}
& 1=2 \int_{\rho_{P} c_{2}}^{\rho_{P} C} \frac{\tilde{\alpha}_{h}\left(x, Q_{t o t}\right)}{\rho_{P}} e^{-\int_{\rho_{P} c_{2}}^{x} \frac{\tilde{\alpha}_{h}\left(s, Q_{t o t}\right)}{\rho_{P}} d s} d x \\
& +4 e^{-J_{1}} \int_{\rho_{P}\left(C+c_{2}\right)}^{2 \rho_{P} C} \frac{\tilde{\alpha}_{h}\left(x, Q_{t o t}\right)}{\rho_{P}} e^{-\int_{\rho_{P}\left(C+c_{2}\right)}^{x} \frac{\tilde{\alpha}_{h}\left(s, Q_{t o t}\right)}{\rho_{P}} d s} d x \\
& +8 e^{-J_{1}-J_{2}} \int_{\rho_{P}\left(2 C+c_{2}\right)}^{1} \frac{\tilde{\alpha}_{h}\left(x, Q_{t o t}\right)}{\rho_{P}} e^{-\int_{\rho_{P}\left(2 C+c_{2}\right)}^{x} \frac{\tilde{\alpha}_{h}\left(s, Q_{t o t}\right)}{\rho_{P}} d s} d x, \\
& =-2\left[e^{-\int_{\rho_{P} c_{2}}^{x} \frac{\tilde{\alpha}_{h}\left(s, Q_{t o t}\right)}{\rho_{P}} d s}\right]_{\rho_{P} c_{2}}^{\rho_{P} C} \\
& -4 e^{-J_{1}}\left[e^{-\int_{\rho_{P}\left(C+c_{2}\right)}^{x} \frac{\tilde{\alpha}_{h}\left(s, Q_{t o t}\right)}{\rho_{P}} d s}\right]_{\rho_{P}\left(C+c_{2}\right)}^{2 \rho_{P} C} \\
& -8 e^{-J_{1}-J_{2}}\left[e^{-\int_{\rho_{P}\left(2 C+c_{2}\right)}^{x} \frac{\tilde{\alpha}_{h}\left(s, Q_{t o t}\right)}{\rho_{P}} d s}\right]_{\rho_{P}\left(2 C+c_{2}\right)}^{1}, \\
& =-2 e^{-J_{1}}+2-4 e^{-J_{1}-J_{2}}+4 e^{-J_{1}}-8 e^{-J_{1}-J_{2}-J_{3}}+8 e^{-J_{1}-J_{2}}, \\
& 0=1+2 e^{-J_{1}}+4 e^{-J_{1}-J_{2}}-8 e^{-J_{1}-J_{2}-J_{3}} .
\end{aligned}
$$

By multiplying the last equation by $e^{J_{1}+J_{2}+J_{3}}$, we obtain the equation:

$$
\varphi\left(Q_{t o t}\right)=0
$$

As $\tilde{\alpha}_{h}$ is strictly decreasing with respect to $Q_{t o t}$, we have that the coefficients $J_{1}, J_{2}$ and $J_{3}$ are strictly decreasing depending with $Q_{t o t}$. This means that $\varphi$ is a strictly decreasing function of $Q_{t o t}$. Furthermore we have $\varphi(\infty)=-1<0$ because $J_{1}=J_{2}=J_{3}=0$ for $Q_{t o t}=+\infty$, and $\varphi(0)>0$ by hypothesis.

According to the intermediate values theorem, continuity of $\varphi$ ensures the existence of a zero in the interval $(0,+\infty)$. The uniqueness of this zero is given by the strict monotonicity of $\varphi$.

One can notice that the steady state for $Q_{t o t}$ does not depend on $\beta_{h}$. The condition $f_{\alpha_{h}}(\infty)=0$ is always satisfied in the model. It reflects saturation of the number of cells in the bone marrow. However it is not optimal, it is possible to transform the proposition into a necessary and sufficient condition assuming $\varphi(\infty)<0$. 
Here we focus on the steady state when leukemic cells populations are all zero. In this case we can write $Q_{t o t}=\int Q_{h}+Q_{h}^{*}$ (at equilibrium).

$$
\begin{aligned}
\int_{0}^{1} Q_{h}(x) d x & =\frac{\beta_{h}}{\rho_{Q}} Q_{h}^{*}\left[1-\int_{0}^{1} \int_{0}^{x} \chi_{1}(y) \frac{\tilde{\alpha}_{h}(y)}{\rho_{P}} \exp \left(-\int_{0}^{y} \frac{\tilde{\alpha}_{h}(s)}{\rho_{P}} d s\right) d y d x\right], \\
& =\frac{\beta_{h}}{\rho_{Q}} Q_{h}^{*}\left[1-\int_{0}^{1}(1-y) \chi_{1}(y) \frac{\tilde{\alpha}_{h}(y)}{\rho_{P}} \exp \left(-\int_{0}^{y} \frac{\tilde{\alpha}_{h}(s)}{\rho_{P}} d s\right) d y\right], \\
& =\frac{\beta_{h}}{\rho_{Q}} Q_{h}^{*} \int_{0}^{1} y \chi_{1}(y) \frac{\tilde{\alpha}_{h}(y)}{\rho_{P}} \exp \left(-\int_{0}^{y} \frac{\tilde{\alpha}_{h}(s)}{\rho_{P}} d s\right) d y .
\end{aligned}
$$

To ease the calculations, we introduce the notation:

$$
L_{1}\left(Q_{t o t}\right)=\int_{0}^{1} y \chi_{1}(y) \frac{\tilde{\alpha}_{h}\left(y, Q_{t o t}\right)}{\rho_{P}} \exp \left(-\int_{0}^{y} \frac{\tilde{\alpha}_{h}\left(s, Q_{t o t}\right)}{\rho_{P}} d s\right) d y .
$$

Since $Q_{t o t}=\int Q_{h}+Q_{h}^{*}$ at equilibrium, we obtain an expression of $Q_{h}^{*}$ based on $Q_{t o t}$ and $P_{t o t}$ :

$$
Q_{h}^{*}=\frac{Q_{t o t}}{1+\frac{\beta_{h}\left(P_{t o t}\right)}{\rho_{Q}} L_{1}\left(Q_{t o t}\right)} .
$$

Using $Q_{h}^{*}$, we can find an equation for the steady state $P_{t o t}$ :

$$
\begin{aligned}
P_{t o t} & =\int_{0}^{1}\left(\chi_{1}(x)+\chi_{2}(x)\right) P(x) d x, \\
& =\frac{\beta_{h}}{\rho_{P}} Q_{h}^{*} \int_{0}^{1}\left(\chi_{1}(x)+\chi_{2}(x)\right) \exp \left(-\int_{0}^{x} \frac{\tilde{\alpha}_{h}(s)}{\rho_{P}} d s\right) d x .
\end{aligned}
$$

Setting

$$
L_{2}\left(Q_{t o t}\right)=\int_{0}^{1}\left(\chi_{1}(x)+\chi_{2}(x)\right) \exp \left(-\int_{0}^{x} \frac{\tilde{\alpha}_{h}\left(s, Q_{t o t}\right)}{\rho_{P}} d s\right) d x,
$$

we then find a condition for the existence and uniqueness of a steady state for $P_{t o t}$.

Proposition 2 Once $Q_{\text {tot }}$ is fixed, equation

$$
P_{t o t}=\frac{\frac{\beta_{h}\left(P_{t o t}\right)}{\rho_{P}} L_{2}\left(Q_{t o t}\right)}{1+\frac{\beta_{h}\left(P_{t o t}\right)}{\rho_{Q}} L_{1}\left(Q_{t o t}\right)} Q_{t o t}
$$

has a unique strictly positive solution $P_{t o t}$.

Proof. We need to show that there is a unique solution to the fixed point equation

$$
x=F(x), \quad x>0
$$


with $F: x \rightarrow \frac{a f(x)}{1+b f(x)}$ where $a$ and $b$ are positive constants, and $f$ is a positive, strictly decreasing differentiable function: $F(0)>0$ and $F^{\prime}(x)=\frac{a f^{\prime}(x)}{(1+b f(x))^{2}}<$ 0 since $f$ is strictly decreasing. Then, the fixed point equation has an unique positive solution.

\subsection{Local stability around the disease-free steady state}

Here we look at the stability of the unique positive leukemic-free steady state $\left(Q_{h}^{e q}, Q_{h}^{* e q}, P_{h}^{e q}, 0,0,0\right)^{T}$ with respect to a small disturbance.

The simplified system IIc linearized around the healthy steady state is:

$$
\left\{\begin{aligned}
\frac{\partial Q_{h}}{\partial t}-\rho_{Q} \frac{\partial Q_{h}}{\partial x}= & \chi_{1}(x) \tilde{\alpha}_{h}\left(x, Q_{t o t}\right) P_{h} \\
& +\chi_{1}(x)\left[\frac{\partial \tilde{\alpha}_{h}}{\partial Q_{t o t}}\left(x, Q_{t o t}\right)\left(Q_{l}+Q_{l}^{*}+Q_{h}+Q_{h}^{*}\right) P_{h}^{e q}\right] \\
= & \rho_{Q} Q_{h}(0)-\beta_{h}\left(0, P_{t o t}\right) Q_{h}^{*}- \\
& \frac{\partial \beta_{h}}{\partial Q_{t o t}}\left(0, P_{t o t}\right)\left(P_{l}+P_{h}\right) Q_{h}^{* e q}, \\
\frac{\partial P_{h}}{\partial t}+\rho_{P} \frac{\partial P_{h}}{\partial x}= & -\left(m+\tilde{\alpha}_{h}\left(x, Q_{t o t}\right)\right) P_{h} \\
& -\frac{\partial \tilde{\alpha}_{h}}{\partial Q_{t o t}}\left(x, Q_{t o t}\right)\left(Q_{l}+Q_{l}^{*}+Q_{h}+Q_{h}^{*}\right) P_{h}^{e q}, \\
\frac{\partial Q_{l}}{\partial t}-\rho_{Q} \frac{\partial Q_{l}}{\partial x}= & \chi_{1}(x) \tilde{\alpha}_{l}\left(x, Q_{t o t}\right) P_{l}, \\
\frac{\partial Q_{l}^{*}}{\partial t}= & \rho_{Q} Q_{l}(0)-\beta_{l}\left(0, P_{t o t}\right) Q_{l}^{*}, \\
\frac{\partial P_{l}}{\partial t}+\rho_{P} \frac{\partial P_{l}}{\partial x}= & -\left(m+\tilde{\alpha}_{l}\left(x, Q_{t o t}\right)\right) P_{l} .
\end{aligned}\right.
$$

The boundary conditions are

$$
\left\{\begin{array}{l}
Q_{l}(t, 1)=0 \\
Q_{h}(t, 1)=0 \\
\rho_{P} P_{l}(t, 0)=\beta_{l}\left(0, P_{t o t}\right) Q_{l}^{*}(t) \\
\rho_{P} P_{h}(t, 0)=\beta_{h}\left(0, P_{t o t}\right) Q_{h}^{*}(t)
\end{array}\right.
$$

The last three differential equations are independent from the first three, the linearized leukemic subsystem is decoupled from the healthy subsystem. We will ignore the healthy cells and study leukemic populations around its steady state $(0,0,0)^{T}$.

The linearized subsystem preserves the positivity of solutions and the dominant real eigenvalue dictates the long-term behavior of solutions. 
We are looking for positive solutions in the form

$$
\left\{\begin{array}{l}
Q_{l}(x, t)=\hat{Q}(x) e^{\lambda t}, \\
Q_{l}^{*}(x)=\hat{Q}^{*} e^{\lambda t} \\
P_{l}(x, t)=\hat{P}(x) e^{\lambda t},
\end{array}\right.
$$

where $\lambda$ is an arbitrary real eigenvalue and $\hat{Q}, \hat{Q}^{*}$ and $\hat{P}$ are associated eigenvectors.

Equations on $\hat{Q}, \hat{P}$ et $\hat{Q}^{*}$ are

$$
\left\{\begin{array}{l}
\lambda \hat{Q}-\rho_{Q} \frac{\partial \hat{Q}}{\partial x}=\chi_{1} \tilde{\alpha}_{l}\left(x, Q_{t o t}\right) \hat{P} \\
\lambda \hat{Q}^{*}=\rho_{Q} \hat{Q}(0)-\beta_{l}\left(0, P_{t o t}\right) \hat{Q}^{*} \\
\lambda \hat{P}+\rho_{P} \frac{\partial \hat{P}}{\partial x}=-\left(m+\tilde{\alpha}_{l}\left(x, Q_{t o t}\right)\right) \hat{P}
\end{array}\right.
$$

To simplify the notations, we remove the hats from eigenvectors. We seek a dominant real eigenvalue $\lambda$, as the differential eigensystem admits positive solutions:

$$
\left\{\begin{array}{l}
\lambda Q-\rho_{Q} \frac{\partial Q}{\partial x}=\chi_{1}(x) \tilde{\alpha}_{l}\left(x, Q_{t o t}\right) P, \\
\lambda P+\rho_{P} \frac{\partial P}{\partial x}=-m P-\tilde{\alpha}_{l}\left(x, Q_{t o t}\right) P
\end{array}\right.
$$

with boundary conditions

$$
\left\{\begin{array}{l}
Q(1)=0 \\
\lambda Q^{*}=\rho_{Q} Q(0)-\beta_{l}\left(0, P_{t o t}\right) Q^{*} \\
\rho_{P} P(0)=\beta_{l}\left(0, P_{t o t}\right) Q^{*}
\end{array}\right.
$$

We explicitly solve this system of ODE, taking into account boundary conditions at $x=0$ :

$$
\left\{\begin{array}{l}
P(x)=\frac{\beta_{l}}{\rho_{P}} Q^{*} \exp \left(-\frac{\lambda+m}{\rho_{P}} x\right) \exp \left(-\int_{0}^{x} \frac{\tilde{\alpha}_{l}(s)}{\rho_{P}} d s\right) \\
Q(x)=\frac{\beta_{l}}{\rho_{Q}} Q^{*} e^{\frac{\lambda}{\rho_{Q}} x}\left[\frac{\lambda+\beta_{l}}{\beta_{l}}-\int_{0}^{x} \chi_{1}(y) e^{-\left(\frac{\lambda+m}{\rho_{P}}+\frac{\lambda}{\rho_{Q}}\right) y} \frac{\tilde{\alpha}_{l}(y)}{\rho_{P}} e^{-\int_{0}^{y} \frac{\tilde{\alpha}_{l}(s)}{\rho_{P}} d s} d y\right]
\end{array}\right.
$$

The boundary condition at $x=1, Q(1)=0$, provides an equation satisfied by $\lambda$ :

Proposition 3 Linearized leukemic subsystem around zero admits a unique real dominant eigenvalue, which is the only real solution of the characteristic equation:

$$
\lambda=\beta_{l}\left[\int_{0}^{1} \chi_{1}(y) e^{-\left(\frac{\lambda+m}{\rho_{P}}+\frac{\lambda}{\rho_{Q}}\right) y} \frac{\tilde{\alpha}_{l}(y)}{\rho_{P}} \exp \left(-\int_{0}^{y} \frac{\tilde{\alpha}_{l}(s)}{\rho_{P}} d s\right) d y-1\right]
$$


The dominant eigenvalue determines the asymptotic behavior of leukemic populations around zero when healthy cell populations are at steady state, and so the asymptotic remission speed in case of effective treatment.

Proof. The right-hand-side is decreasing as a function of $\lambda$, while the left-handside is increasing from $-\infty$ to $+\infty$. Thus there is one, and only one real solution $\lambda$ to this equation.

Let $z$ be a complex solution, not real.

$$
\begin{aligned}
\Re(z) & =\beta_{l}\left[\Re\left(\int_{0}^{1} \chi_{1}(y) e^{-\left(\frac{z+m}{\rho_{P}}+\frac{z}{\rho_{Q}}\right) y} \frac{\tilde{\alpha}_{l}(y)}{\rho_{P}} e^{-\int_{0}^{y} \frac{\tilde{\alpha}_{l}(s)}{\rho_{P}} d s} d y\right)-1\right] \\
& <\beta_{l}\left[\left|\int_{0}^{1} \chi_{1}(y) e^{-\left(\frac{z+m}{\rho_{P}}+\frac{z}{\rho_{Q}}\right) y} \frac{\tilde{\alpha}_{l}(y)}{\rho_{P}} e^{-\int_{0}^{y} \frac{\tilde{\alpha}_{l}(s)}{\rho_{P}} d s} d y\right|-1\right] \\
& <\beta_{l}\left[\int_{0}^{1} \chi_{1}(y) e^{-\left(\frac{\Re(z)+m}{\rho_{P}}+\frac{\Re(z)}{\rho_{Q}}\right) y} \frac{\tilde{\alpha}_{l}(y)}{\rho_{P}} e^{-\int_{0}^{y} \frac{\tilde{\alpha}_{l}(s)}{\rho_{P}} d s} d y-1\right]
\end{aligned}
$$

So $\Re(z)$ satisfies the inequality case of the characteristic equation. Since the right term of this inequality is decreasing with $\Re(z), \Re(z)$ is necessary lower than $\lambda$, solution of the characteristic equation.

The flux of differentiated cells $D$ is given by the formula $D(t)=\rho_{P} P(t, 1)$, so it admits the same asymptotic behavior as $P$. It follows that the quantity of mature leukemic cells in blood also behaves like $e^{\lambda t}$. As the number of healthy mature cells in the blood (computed with the formula (3)) converges to a positive constant, the BCR-ABL/ABL ratio predicted by the model around the diseasefree steady state behaves asymptotically as $e^{\lambda t}$. Therefore, the solution of the characteristic equation (10) can be interpreted as the asymptotic growth (if positive) or remission (if negative) rate of the disease in the blood. Independently of the healthy population stability, characterizing how $\lambda$ behaves with and without treatment is crucial. In particular we want to show when $\lambda$ is negative.

Knowledge of the function $f_{\alpha_{h}}$ gives the value of $Q_{t o t}$ at equilibrium. We can find $P_{t o t}$ based on $Q_{t o t}$ and $f_{\beta_{h}}$. Then transfer rates $\alpha_{l}$ and $\beta_{l}$ can be computed, based on $f_{\alpha_{l}}, f_{\beta_{l}}, Q_{t o t}$ and $P_{t o t}$. We finally deduce the value of $\lambda$, based on $\alpha_{l}, \beta_{l}$ and $m$. This way, we can determine the speed of asymptotic decay of the $\mathrm{BCR}-\mathrm{ABL} / \mathrm{ABL}$ ratio under treatment, according to the parameters of the problem.

According to the model, if the dominant eigenvalue $\lambda$ is negative, the treatment works; the leukemic cell number goes to zero. Otherwise, it means that the disease-free steady state is unstable, so that treatment can not eradicate CML.

\section{Treatment}

\subsection{Treatment effects}

Glauche et al. (2012) study the effect of TKI treatment in Roeder model. They model TKI effect by the death of a constant percentage of leukemic proliferating 
cells per unit time (death rate $m=3.2 \%$ per hour (Roeder et al. 2006)), and by reducing the leukemic quiescent cells activation rate $\left(\beta_{l}\right.$ lower). TKI treatments are supposed to affect only leukemic stem cells (Figure 5).

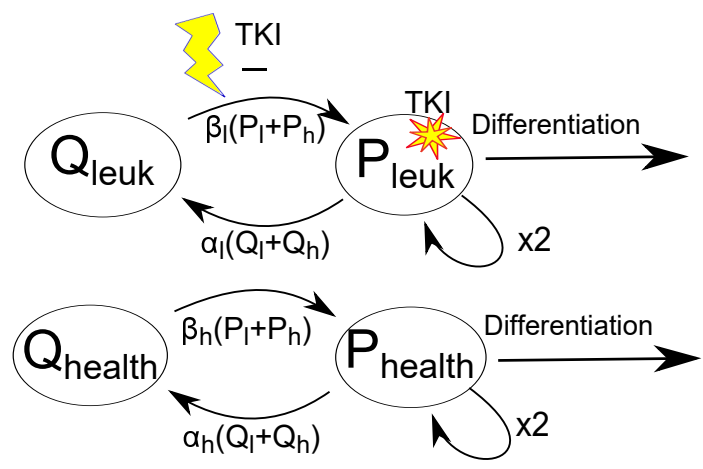

Fig. 5. Action of TKI on the Roeder et al. model. TKI treatment is supposed to affect only leukemic stem cells, by killing proliferative ones and preventing quiescent ones from activation.

We have shown in the previous section that a unique real eigenvalue $\lambda$ drives the long-term dynamics of the leukemic populations. Here we look at how $\lambda$ varies when treatment is taken into account.

Only some of the parameters are affected by the type of treatment considered. It is thought that TKI treatments affect the proliferative cell death rate $m$ and the activation rate of the quiescent leukemic cells through the function $f_{\beta_{l}}$. TKIdependent parameters were estimated from experimental clinical data, obtained on patients treated with a constant dose of TKI (Roeder et al. 2006). Parameters values without and with treatment are given in Table 1 . We will parameterize the death rate $m$ as $m(\xi)$ such that $m(0)=m_{0}$ and $m(1)=m_{1}$, where $m_{0}$ and $m_{1}$ are the death rates without and with treatment respectively. In the same way, we will parameterize activation rate $\beta\left(P_{t o t}\right)$ as $\beta\left(P_{t o t} ; \xi\right)$ such that $\beta\left(P_{t o t} ; 0\right)=\beta_{0}\left(P_{t o t}\right)$ and $\beta\left(P_{t o t} ; 1\right)=\beta_{1}\left(P_{t o t}\right)$, where $\beta_{0}$ and $\beta_{1}$ are the activation rates without and with treatment for leukemic cells respectively. The simplest way to proceed is to assume a linear dependence of the death rate

$$
m(\xi)=m_{0}+\left(m_{1}-m_{0}\right) \xi
$$

When the drug has only one target, dose-response curves are typically monotonous: the effect increases as the dose is increased. Here, the treatment has two non-additive effects that can influence efficacy: increase in the proliferative cell death rate and decrease in the activation rate of quiescent cells. In this section, even if it is not biologically relevant, we allow heuristically the treatment dose $\xi$ to become infinite. An infinite dose corresponds to an infinite death rate of proliferative cells and to the activation rate vanishing. In this situation all 
proliferative cells vanish but quiescent cells stay safe from treatment, so we expect the growth rate to vanish $(\lambda(\xi=\infty)=0)$. As the treatment is assumed to be non-effective at dose 0 and $+\infty$, and effective for at least one positive dose, this means that the growth rate $\lambda$ could not be monotonous.

\subsection{General dependence}

Based on the linear stability analysis performed in Section 3.2, we can re-express the characteristic equation 10 as follows. Let

$$
\Gamma(\lambda, \xi)=\int_{0}^{1} \chi_{1}(x) e^{-\left(\frac{\lambda+\xi m_{1}}{\rho_{P}}+\frac{\lambda}{\rho_{Q}}\right) x} \tilde{\alpha}_{l}(x) / \rho_{P} e^{-\int_{0}^{x} \tilde{\alpha_{l}} / \rho_{P} d s} d x .
$$

The characteristic equation (10) becomes

$$
\lambda(\xi)=\beta(\xi)(\Gamma(\lambda(\xi), \xi)-1) .
$$

To keep the notation simple, we used the fact that $m_{0}=0$.

We propose a wide class of function $\beta$ that leads to a non-monotonous growth rate $\lambda$.

Lemma 1 If $\beta$ is a positive function that decreases towards zero, then $\lambda$ is nonmonotonous and converges to 0 from below when $\xi$ approaches $\infty$.

Proof. To simplify calculations we denote:

$$
\left\{\begin{array}{l}
\Gamma(\lambda, \xi)=\int_{0}^{1} f(\lambda, \xi, x) d x, \\
M_{1}(\lambda, \xi)=\int_{0}^{1} x f(\lambda, \xi, x) d x, \\
M_{2}(\lambda, \xi)=\int_{0}^{1} x^{2} f(\lambda, \xi, x) d x .
\end{array}\right.
$$

Furthermore, we can compute easily,

$$
\left\{\begin{array}{l}
\partial_{\lambda} \Gamma=-\left(\frac{1}{\rho_{P}}+\frac{1}{\rho_{Q}}\right) M_{1}<0, \\
\partial_{\xi} \Gamma=-\frac{m_{1}}{\rho_{P}} M_{1}<0, \\
\partial_{\xi}^{2} \Gamma=\left(\frac{m_{1}}{\rho_{P}}\right)^{2} M_{2}>0, \\
\partial_{\xi} \partial_{\lambda} \Gamma=\left(\frac{1}{\rho_{P}}+\frac{1}{\rho_{Q}}\right) \frac{m_{1}}{\rho_{P}} M_{2}>0, \\
\partial_{\lambda}^{2} \Gamma=\left(\frac{1}{\rho_{P}}+\frac{1}{\rho_{Q}}\right)^{2} M_{2}>0 .
\end{array}\right.
$$

The characteristic equation (11) is uniquely solvable and regularity of $\lambda$ is obtained by classical implicit function theorem. By differentiating with respect to $\xi$, we find:

$$
\lambda^{\prime}=\beta^{\prime}(\Gamma-1)+\beta \partial_{\xi} \Gamma+\lambda^{\prime} \beta \partial_{\lambda} \Gamma, \text { that is to say, } \quad \lambda^{\prime}=\frac{\beta^{\prime}(\Gamma-1)+\beta \partial_{\xi} \Gamma}{1-\beta \partial_{\lambda} \Gamma} .
$$


Then

$$
\lambda^{\prime}=\frac{\frac{\beta^{\prime}}{\beta} \lambda-\beta \frac{m_{1}}{\rho_{P}} M_{1}}{1+\beta\left(\frac{1}{\rho_{P}}+\frac{1}{\rho_{Q}}\right) M_{1}} .
$$

If $\lambda$ is positive, as $\beta$ is decreasing, $\lambda^{\prime}<0$. So, the function $\lambda$ is decreasing as soon as $\lambda \geqslant 0$. If $\lambda(0) \geqslant 0, \lambda$ stays bounded by $\lambda(0)$. In this case, since $\Gamma$ is positive and is decreasing with $\lambda$, we can write:

$$
-\beta(\xi) \leqslant \lambda(\xi) \leqslant \beta(\xi)(\Gamma(\lambda(0), \xi)-1)
$$

Since $\Gamma(\lambda(0), \xi)$ converges to 0 when $\xi$ approaches $\infty$, the second right inequality of (13) assures that $\lambda$ becomes negative in finite time and stays negative until $\infty$. Then, as $\beta$ converges to 0 when $\xi$ approaches $\infty$, the left inequality of (13) assures that $\lambda$ converges to 0 from below when $\xi$ approaches $\infty$.

The above calculation (equation (12)) also gives that $\left.\lambda^{\prime}\right|_{\lambda=0}<0$. This inequality ensures that, once negative, $\lambda$ can not become positive again. It remains strictly negative from a certain value of $\xi$, denoted $\xi_{1}$. So the result stays true if $\lambda(0)<0$.

The growth rate $\lambda$ therefore is non-monotonous and allows a minimum at $\xi_{2} \in\left(\xi_{1},+\infty\right)$.

Proposition 4 If $m$ is linear of the form $m(\xi)=m_{1} \xi$, and if $\beta$ is a positive log-concave function that decreases towards zero, then there is a unique $\xi$ that minimizes $\lambda$.

Proof. We keep the notations of the above lemma and we set $B=\log \beta$ and $g=B^{\prime} \lambda-\beta m_{1} M_{1} / \rho_{P}$, so that $\lambda^{\prime}=\frac{g}{d}$ where $d$ is a strictly positive function (equation (12)). To determine the sign of $\lambda$, it is sufficient to study function $g$.

$$
\begin{aligned}
g^{\prime} & =B^{\prime \prime} \lambda+B^{\prime} \lambda^{\prime}-m_{1} / \rho_{P}\left(\beta^{\prime} M_{1}+\beta\left(\lambda^{\prime} \partial_{1} M_{1}+\partial_{2} M_{1}\right)\right) \\
& =B^{\prime \prime} \lambda+B^{\prime} \lambda^{\prime}-m_{1} / \rho_{P} \beta^{\prime} M_{1}+m_{1} / \rho_{P} \beta\left(\lambda^{\prime}\left(\frac{1}{\rho_{P}}+\frac{1}{\rho_{Q}}\right) M_{2}+m_{1} / \rho_{P} M_{2}\right) \\
& =B^{\prime \prime} \lambda+B^{\prime} \lambda^{\prime}-m_{1} / \rho_{P} \beta^{\prime} M_{1}+m_{1} / \rho_{P} \beta M_{2}\left(\lambda^{\prime}\left(\frac{1}{\rho_{P}}+\frac{1}{\rho_{Q}}\right)+m_{1} / \rho_{P}\right)
\end{aligned}
$$

By assumption on $\beta$, the first and second derivatives $B^{\prime}$ and $B^{\prime \prime}$ are negative. In addition, $\beta^{\prime}$ is negative and $M_{2}$ is strictly positive. Moreover we stand in the interval $\left(\xi_{1} ;+\infty\right)$, so $\lambda$ is strictly negative. If $\lambda^{\prime}(\xi)=0$ for a given $\xi$, we have $g(\xi)=0$ and $g^{\prime}(\xi)>0$, so $g$ is strictly positive on an interval of the form $(\xi ; \xi+\varepsilon)$. We deduce that $\lambda$ can not be locally constant.

We consider now the case where $\lambda^{\prime}$ is positive. We can write $g^{\prime}=B^{\prime} \lambda^{\prime}+K$, where $K$ is a positive function. We have $\left[\lambda^{\prime} d\right]^{\prime}=g^{\prime}=B^{\prime} \lambda^{\prime}+K=\frac{B^{\prime}}{d} \lambda^{\prime} d+$ $K>\frac{B^{\prime}}{d} \lambda^{\prime} d$. As $B^{\prime \prime} \leqslant 0, B^{\prime}$ is decreasing. In particular, for every $\xi>\xi_{2}$, 
$B^{\prime}(\xi) \leqslant B^{\prime}\left(\xi_{2}\right)$. Similarly, for any $\xi>\xi_{2}, \beta(\xi) \leqslant \beta\left(\xi_{2}\right)$. Since $\lambda$ is assumed to be strictly negative, $\Gamma<1$. And, as $M_{1}<\Gamma$, we can set an upper bound on function $d$ with the constant $1+\beta\left(\xi_{2}\right)\left(\frac{1}{\rho_{P}}+\frac{1}{\rho_{Q}}\right)$.

We denote $c=\frac{-B^{\prime}\left(\xi_{2}\right)}{1+\beta\left(\xi_{2}\right)\left(\frac{1}{\rho_{P}}+\frac{1}{\rho_{Q}}\right)}>0$. We then have $\left[\lambda^{\prime} d\right]^{\prime}>-c \lambda^{\prime} d$.

Let $\xi_{3}>\xi_{2}$ be such that $\lambda^{\prime}\left(\xi_{3}\right)>0$. According to Gronwall Lemma,

$$
\forall \xi \geqslant \xi_{3}, \lambda^{\prime}(\xi) d(\xi) \geqslant \lambda^{\prime}\left(\xi_{3}\right) d\left(\xi_{3}\right) e^{-c \xi} .
$$

As $d$ is a positive function and $\lambda^{\prime}\left(\xi_{3}\right)>0$, we obtain $\forall \xi \geqslant \xi_{3}, \lambda^{\prime}(\xi)>0$.

we have shown that $\lambda$ is strictly decreasing and reaches a negative local minimum at $\xi=\xi_{2}$, and that once it is increasing it is increasing on all intervals of the type $\left(\xi_{3},+\infty\right)$. Since it can not be stationary, it must grow exactly on $\left(\xi_{2},+\infty\right)$, and therefore $\xi_{2}$ is the unique minimum of $\lambda$ on $\mathbb{R}_{+}$.

That kind of convexity result was treated in more general context (Clairambault et al. 2011).

\subsection{Examples of dose-dependence parameters}

The modeling of treatment dose dependence on parameters is an open question. While it seems intuitive to model a death rate by a linear function of dose, it is not well understood how treatment dose could affect a transition rate. However we proved in Proposition 4 that our result stands for a large class of functions modeling the activation rate $\beta_{l}$. In order to illustrate our result, we choose to study two of them; a linear and a exponential dependence of the activation rate $\beta_{l}$. In fact, both can verify the conditions of the above Proposition 4 . We denote by $\beta_{0}$ the activation rate without treatment (dose 0 in Table 1), $m_{1}$ and $\beta_{1}$ the death rate and the activation rate with treatment (dose 1 in Table 1).

We computed the dose-response curve $\lambda($ dose) using the complete model, the simplified model, or the characteristic equation (Figure 6 and 7). The doseresponse curves we obtained are not monotonous. This is unexpected since it means that applied too much treatment is detrimental to its efficacy against the disease, according to the model. Indeed there is a unique finite dose that minimizes the value of $\lambda$, and therefore maximizes the long-term efficacy of treatment with TKI.

Linear dose-dependence for activation rate (Figure 6) We linearly adjust the activation rate depending on the TKI dose from 0 (no treatment) to 1 (fulldose treatment, according to Roeder et al. (2006)). For a dose higher than 1 we choose a smooth connection to 0 in order to respect Proposition 4 hypothesis. We set

$$
\left\{\begin{array}{l}
\beta(\xi)=\beta_{0}-\left(\beta_{0}-\beta_{1}\right) \xi \text { if } \xi \in[0,1] \\
\beta(\xi)=\beta_{1} \exp \left(-\frac{\beta_{0}-\beta_{1}}{\beta_{1}}(\xi-1)\right) \text { if } \xi>1
\end{array}\right.
$$


Function $\beta$ is continuous, and two times differentiable with respect to $\xi$, and log-concave. So it verifies Proposition 4 hypothesis.



Fig. 6. Dependence of the asymptotic growth according to the TKI treatment intensity. The theoretical curve represents eigenvalue of system linearized around 0 , given by the characteristic equation, while simulation curves represent the logarithmic derivative of solutions near to disease-free steady state. Effect of TKI dose is modeled as $\beta(\xi)=\max \left(0, \beta_{0}+\xi\left(\beta_{1}-\beta_{0}\right)\right)$ and $m(\xi)=\xi m_{1}$, where $\beta_{0}$ is the activation rate without treatment, $m_{1}$ and $\beta_{1}$ are the death rate and the activation rate with treatment (corresponding to the standard dose, according to Roeder et al. (2006)).

Exponential dose-dependence for activation rate (Figure 7 ). In more realistic settings $\beta(\xi)$ depends geometrically on the dose $\xi$, of the form $\beta(\xi)=$ $\beta_{1}^{\xi} \beta_{0}^{1-\xi}$. This transition rate $\beta$ verifies $\log (\beta)^{\prime \prime}=0$, so it represents the limit case of Proposition 4.

\subsection{Comparison with standard parameters.}

Figures 6 and 7 highlight the idea that there could be optimal treatments at doses lower than the one used by default (Roeder et al. 2006). Using these parameters, both cases leads to non-monotonous function $\lambda(\xi)$, with a minimum that occurs for a dose lower than 1 . Here, we show that it is a more general result, using numerical simulations with standard parameters.

Proposition 5 If the minimum of $\lambda$ occurs at a dose lower than 1 in the case where $\beta(\xi)=\beta_{1}^{\xi} \beta_{0}^{1-\xi}$, then for any log-concave positive and decreasing to zero parametrization of $\beta$ that satisfies $\beta(0)=\beta_{0}$ and $\beta(1)=\beta_{1}$, the minimum of $\lambda$ occurs at a dose lower than 1. 




Fig. 7. Dependence of the asymptotic growth according to the TKI treatment intensity. The theoretical curve represents eigenvalue of system linearized around 0 , given by the characteristic equation, while simulation curves represent the logarithmic derivative of solutions near to disease-free steady state. Effect of TKI dose is modeled as $\beta(\xi)=$ $\beta_{1}^{\xi} \beta_{0}^{1-\xi}$ and $m(\xi)=\xi m_{1}$, where $\beta_{0}$ is the activation rate without treatment, $m_{1}$ and $\beta_{1}$ are the death rate and the activation rate with treatment (corresponding to the standard dose, according to Roeder et al. (2006))

Proof. We denote $\bar{\beta}(\xi)=\beta_{1}^{\xi} \beta_{0}^{1-\xi}$. Numerically, this exponential parametrization leads to a a minimum for $\bar{\lambda}$ that occurs for a dose lower than 1 (Figure 7 ).

Let $\beta$ be a log-concave parametrization verifying $\beta(0)=\beta_{0}$ and $\beta(1)=\beta_{1}$. As $\beta$ is log-concave,

$$
\begin{aligned}
\frac{\beta^{\prime}(1)}{\beta(1)}=(\log (\beta))^{\prime}(1) \leqslant \frac{\log (\beta(1))-\log (\beta(0))}{1-0} & =\frac{\log \left(\beta_{1}\right)-\log \left(\beta_{0}\right)}{1-0} \\
& =(\log (\bar{\beta}))^{\prime}(1) \\
& =\frac{\bar{\beta}^{\prime}(1)}{\bar{\beta}(1)}
\end{aligned}
$$

Then, as $\lambda(1)=\bar{\lambda}(1)<0$,

$$
\lambda^{\prime}(1)=\frac{\frac{\beta^{\prime}(1)}{\beta(1)} \lambda(1)-\beta \frac{m_{1}}{\rho_{P}} M_{1}}{1+\beta\left(\frac{1}{\rho_{P}}+\frac{1}{\rho_{Q}}\right) M_{1}} \geqslant \frac{\frac{\bar{\beta}^{\prime}(1)}{\bar{\beta}(1)} \bar{\lambda}(1)-\bar{\beta} \frac{m_{1}}{\rho_{P}} M_{1}}{1+\bar{\beta}\left(\frac{1}{\rho_{P}}+\frac{1}{\rho_{Q}}\right) M_{1}}=\bar{\lambda}^{\prime}(1) .
$$

We have shown that if $\bar{\lambda}^{\prime}(1)>0$, then $\lambda^{\prime}(1)>0$. So, the existence of a minimum in $(0,1)$ for $\bar{\lambda}$ induces the existence of a minimum in $(0,1)$ for $\lambda$.

Numerically, we stand in the case of Proposition 5. So we have shown that, for a large class of functions modeling the treatment effect on activation rate, the asymptotic decay is not monotonous, and admits a minimum at a dose smaller than 1, corresponding to the standard dose, according to Roeder et al. (2006). 


\section{Discussion and conclusion}

We proposed a simplification of the Roeder et al. model. The formulation of the model as a PDE system makes it possible to simulate solutions much faster than with an ABM, and to consider the cell populations as continuous time-dependent densities. In addition, we have seen numerically, that the simplification of the PDE system by partial integration and the restriction of the cell densities on well-chosen characteristics, maintained the behavior of the solutions. However the simplification could be less relevant for studying other scenarios, such as combination or pulsed therapies or for exploring short-term effects. The simplification allowed the analysis of the long-term dynamics of the system. We proved the existence and uniqueness of a healthy steady state, then the existence and uniqueness of a real dominant eigenvalue for this steady state. We then investigated the dependence of this eigenvalue on the treatment dose, and we found a condition for this dependence not to be monotonous. The simplification we made is weaker than the one made by Doumic-Jauffret et al. (2010), and the analysis remains feasible. In addition, on the basis of their work, we could study the dependence of the response depending on other parameters, and draw bifurcation graph and stability regions. It was anticipated that the simplified model would possess an unique positive disease-free steady states. It corresponds to the asymptotic solution when treatment is effective. Whether leukemic cell pool completely vanishes under treatment is now a biological open question (Chu et al. 2011, Ross et al. 2010). Our analysis of the remission rate (decay rate of leukemic cell populations) yielded an unexpected result about treatments using tyrosine kinase inhibitors. Figures 6 and 7 highlight the idea that there could be optimal treatments at doses lower than the one used by default (Roeder et al. 2006). This counter-intuitive result is not due to the simplification of the PDE model; it is possible to observe the same phenomenon for full model. According to this idea, decreasing default TKI dose could improve the long-term efficacy, while increasing it could slow down the remission rate. The natural dynamics have been rather the reverse.

The results we have obtained are about long-term dynamics that are not correlated to short-term dynamics. The optimal long-term treatment dose we have identified probably does not ensure an optimal short-term response in a clinical setting. Rather it could improve the remission speed of patients who already reached a low level of leukemic cells.

Here, we consider a single leukemic clone. It could be interesting to deal with several clones (Stiehl et al. n.d., Valent 2008), with different treatment sensitivity, for instance. Our results would apply to each clone individually.

The Roeder et al. model provides results that are coherent with clinical data. However, it remains too complex to provide theoretical results on cellular dynamics. The simplification we have introduced allows a mathematical analysis and provides results on asymptotic dynamics. The non monotonous effect of TKI treatment suggests the existence of an optimal treatment strategy, by balancing the cytotoxic and the antiproliferative effects of TKIs. This strategy could be clinically testable since it induces a treatment dose reduction, which is patient- 


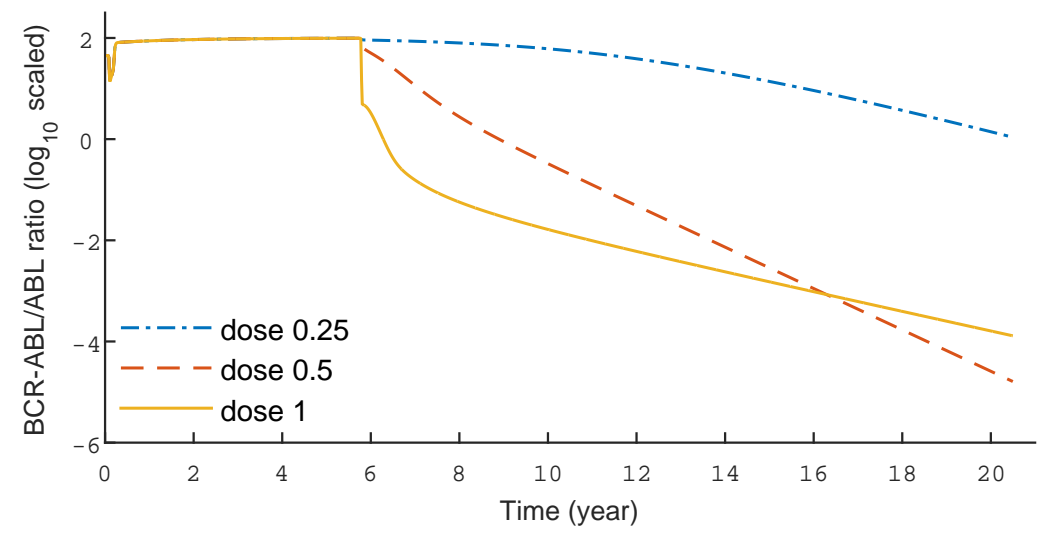

Fig. 8. Progress of the BCR-ABL/ABL ratio over time, for three TKI treatment doses $(0.25,0.5$ and 1$)$. Doses 0.25 and 1 correspond to approximately the same asymptotic decay. Dose 0.5 corresponds approximately to the highest asymptotic decay.

friendly as it decreases the occurrence of side effects (Figure 8). It remains coherent with clinical results on TKI dose (Breccia et al. 2010, Hehlmann et al. 2014).

Glauche et al. (2012) simulated different drug combination regimens and found that alternating the treatment between TKI and interferon alpha leads to a better long-term treatment than TKI alone. Our result suggest that a better response could be obtained by a reduced TKI dose alone. Alternating treatments are expected to be even more effective by allowing quiescent cells to be periodically activated. The existence of a mechanism in TKI action against leukemia that allows a non-monotonous dose response curve opens up interesting perspective for treatment optimization by drug combination and dose adjustment. 


\section{Bibliography}

Breccia M., Cannella, L., Stefanizzi, C. et al (2010), 'Cytogenetic and molecular responses in chronic phase chronic myeloid leukaemia patients receiving low dose of imatinib for intolerance to standard dose', Hematol. Oncol. 28, 89-92.

Chu, S., McDonald, T., Lin, A., Chakraborty, S., Huang, Q., Snyder, D. S. \& Bhatia, R. (2011), 'Persistence of leukemia stem cells in chronic myelogenous leukemia patients in prolonged remission with imatinib treatment', Blood 118(20), 5565-5572.

Clairambault, J., Gaubert, S. \& Lepoutre, T. (2011), 'Circadian rhythm and cell population growth', Math. Comput. Model. 53(7-8), 1558-1567.

Clapp, G. D., Lepoutre, T., El Cheikh, R., Bernard, S., Ruby, J., LabussièreWallet, H., Nicolini, F. E. \& Levy, D. (2015), 'Implication of the autologous immune system in BCR-ABL transcript variations in chronic myelogenous leukemia patients treated with imatinib', Cancer Res. 75(19), 4053-4062.

Deininger, M. W. N., Goldman, J. M. \& Melo, J. V. (2008), 'The molecular biology of chronic myeloid leukemia', Blood 96(10), 3343-3356.

Doumic-Jauffret, M., Kim, P. S. \& Perthame, B. (2010), 'Stability Analysis of a Simplified Yet Complete Model for Chronic Myelogenous Leukemia', Bull. Math. Biol. 72(7), 1732-1759.

Foo, J., Drummond, M. W., Clarkson, B., Holyoake, T. L., Michor, F. (2009), 'Eradication of Chronic Myeloid Leukemia Stem Cells: A Novel Mathematical Model Predicts No Therapeutic Benefit of Adding G-CSF to Imatinib', PLoS Comput. Biol. 5(9), e1000503.

Glauche, I., Horn, K., Horn, M., Thielecke, L., Essers, M. A. G., Trumpp, A. \& Roeder, I. (2012), 'Therapy of chronic myeloid leukaemia can benefit from the activation of stem cells: simulation studies of different treatment combinations.', Br. J. Cancer 106(11), 1742-52.

Graham, S. M., Jorgensen, H. G., Allan, E. K., Pearson, C., Alcorn, M. J., Richmond, L. \& Holyoake, T. L. (2002), 'Primitive, quiescent, Philadelphiapositive stem cells from patients with chronic myeloid leukemia are insensitive to STI571 in vitro', Blood 99(1), 319-325.

Hehlmann, R., Müller, M. C., Lauseker, M. et al (2014), 'Deep molecular response is reached by the majority of patients treated with imatinib, predicts survival, and is achieved more quickly by optimized high-dose imatinib: Results from the randomized CML-Study IV', J. Clin. Oncol. 32(5), 415-423.

Kim, P. S., Lee, P. P. \& Levy, D. (2008a), 'A PDE model for imatinib-treated chronic myelogenous leukemia', Bull. Math. Biol. 70(7), 1994-2016.

Kim, P. S., Lee, P. P. \& Levy, D. (2008b), 'Dynamics and potential impact of the immune response to chronic myelogenous leukemia', PLoS Comput. Biol. 4(6), e1000095.

Kim, P. S., Lee, P. P. \& Levy, D. (2008c), 'Modeling imatinib-treated chronic myelogenous leukemia: Reducing the complexity of agent-based models', Bull. Math. Biol. 70(3), 728-744. 
Komarova, N. L. \& Wodarz, D. (2007), 'Effect of cellular quiescence on the success of targeted CML therapy', PLoS One 2(10), e990

Mahon, F. X., Réa, D., Guilhot, J. et al (2010), 'Discontinuation of imatinib in patients with chronic myeloid leukaemia who have maintained complete molecular remission for at least 2 years: the prospective, multicentre Stop Imatinib (STIM) trial', Lancet Oncol. 11(11), 1029-1035.

Michor, F., Hughes, T. P., Iwasa, Y., Branford, S., Shah, N. P., Sawyers, C. L. \& Nowak, M. A. (2005), 'Dynamics of chronic myeloid leukaemia', Nature 435(June), 1267-1270.

O'Brien, S. G., Guilhot, F., Larson, R. A. et al (2003), 'Imatinib compared with interferon and low-dose cytarabine for newly diagnosed chronic-phase chronic myeloid leukemia.', N. Engl. J. Med. 348(11), 994-1004.

Preudhomme, C., Guilhot, J., Nicolini, F. E. et al (2010), 'Imatinib plus peginterferon alfa-2a in chronic myeloid leukemia.', N. Engl. J. Med. 363(26), 2511-21.

Roeder, I., Herberg, M. \& Horn, M. (2009), "An "age"-structured model of hematopoietic stem cell organization with application to chronic myeloid leukemia', Bull. Math. Biol. 71(3), 602-626.

Roeder, I., Horn, M., Glauche, I., Hochhaus, A., Mueller, M. C. \& Loeffler, M. (2006), 'Dynamic modeling of imatinib-treated chronic myeloid leukemia: functional insights and clinical implications.', Nat. Med. 12(10), 1181-1184.

Ross, D. M., Branford, S., Seymour, J. F. et al (2010), 'Patients with chronic myeloid leukemia who maintain a complete molecular response after stopping imatinib treatment have evidence of persistent leukemia by DNA PCR.', Leuk. Off. J. Leuk. Soc. Am. Leuk. Res. Fund, U.K 24(10), 1719-1724.

Rousselot, P., Huguet, F., Rea, D., Legros, L., Cayuela, J. M., Maarek, O., Blanchet, O., Marit, G., Gluckman, E., Reiffers, J., Gardembas, M. \& Mahon, F. X. (2007), 'Imatinib mesylate discontinuation in patients with chronic myelogenous leukemia in complete molecular remission for more than 2 years', Blood 109(1), 58-60.

Stiehl, T., Baran, N., Ho, A. D. \& Marciniak-czochra, A. (2014 ), 'Clonal selection and therapy resistance in acute leukaemias : mathematical modelling explains different proliferation patterns at diagnosis and relapse'. J.R. Soc. Interface 11(94) 20140079.

Valent, P. (2008), 'Emerging stem cell concepts for imatinib-resistant chronic myeloid leukaemia: Implications for the biology, management, and therapy of the disease', Br. J. Haematol. 142(3), 361-378. 